\title{
RESOLUBILIDADE NA ESTRATÉGIA SAÚDE DA FAMÍLIA DE UM MUNICÍPIO BAIANO: O OLHAR DOS USUÁRIOS
}

\author{
RESOLUBILITY IN THE FAMILY HEALTH STRATEGY OF A MUNICIPALITY IN \\ BAHIA: THE USERS' PERSPECTIVE
}
Geane da Silva Lima ${ }^{1}$, Mariana de Oliveira Araújo ${ }^{2}$, Bianca de Oliveira Araújo ${ }^{2}$, Silvana Gomes Nunes Piva ${ }^{3}$

Enfermeira da Policlínica Regional - Região de Saúde de Senhor do Bonfim ${ }^{1}$; Universidade Estadual de Feira de Santana (UEFS) ${ }^{2}$ e Universidade do Estado da Bahia (UNEB) ${ }^{3}$

\section{Abstract}

The aim of this study was to understand the meaning (s) of resolvability in health care from the perspective of users of the Family Health Strategy (FHS) in a municipality in Bahia and to identify possible difficulties (s) and / or facility (s) found by them, to solve the health problem they present. This is a qualitative survey, which was carried out with 10 users. The data collection techniques were semi-structured interviews and systematic observation, and the data were analyzed using the Thematic Content Analysis method. The results showed that the meaning of resolvability for users is related to their needs, experiences and expectations. The promotion of resolutive actions involves facilities that are related to the actions developed by the professionals for their guarantee and the geographical location of the unit. The difficulties are related to the delay in receiving the results of exams, getting assistance from the medical professional, the absence of medicines at the basic pharmacy, the geographical distance of the Family Health Unit (USF) in relation to their homes, among others. It was concluded that the understanding of the meaning of resolvability in the perception of users, as well as the difficulties and / or facilities that permeate their guarantee is of paramount importance for the promotion of more appropriate interventions developed by health professionals and managers and the quality of the services. services available.

Keywords: Family Health Strategy; Problem Solving; Health Care.

\section{Resumo}

Objetivou-se com este estudo compreender o(s) significado(s) da resolubilidade na assistência à saúde na perspectiva dos usuários da Estratégia Saúde da Família (ESF) de um município baiano e identificar a(s) possíveis dificuldade(s) e/ou facilidade(s) encontrada(s) pelos mesmos, para a resolubilidade do problema de saúde que apresenta. Esta é uma pesquisa qualitativa, que foi realizada com 10 usuários. As técnicas de coleta de dados foram a entrevista semiestruturada e a observação sistemática, sendo os dados analisados com o método de Análise de Conteúdo Temática. Os resultados demonstraram que o significado de resolubilidade para os usuários está relacionado às necessidades, vivências e expectativas que estes apresentam. A promoção de ações resolutivas perpassa por facilidades que estão relacionadas às ações desenvolvidas pelos profissionais para a sua garantia e a localização geográfica da unidade. As dificuldades dizem respeito a demora em receber o resultado de exames, conseguir atendimento com 0 profissional médico, ausência de medicamentos na farmácia básica, a distância geográfica da Unidade de Saúde da Familia (USF) em relação aos seus domicílios, entre outros. Concluiu-se que a compreensão do significado de resolubilidade na percepção dos usuários, bem como das dificuldades e/ ou facilidades que permeiam a sua garantia é de suma importância para a promoção de intervenções mais adequadas desenvolvidas pelos profissionais de saúde $e$ gestores e a qualidade dos serviços disponibilizados.

Palavras-chave: Estratégia Saúde da Família; Resolução de Problemas; Atenção à Saúde. 


\section{Introdução}

O Ministério da Saúde entende que a Estratégia Saúde da Família (ESF) constitui-se em prioritária com vistas à reorganização da Atenção Básica no país, a partir da garantia dos preceitos do Sistema Único de Saúde (SUS), sendo considerada uma estratégia de expansão, qualificação e fortalecimento deste nível de atenção, por possibilitar uma reorientação do processo de trabalho, com potencial de ampliar o acesso e a resolubilidade, bem como impactar positivamente na situação de saúde das pessoas e coletividades ${ }^{1}$.

O Programa Saúde da Família (PSF) surgiu em 1993, influenciado por outros programas já existentes, como o Programa de Agentes Comunitários de Saúde (PACS), o qual buscava enfrentar os graves índices de morbimortalidade materna e infantil na Região Nordeste do país, sendo oficializado em 1994 pelo Ministério da Saúde enquanto instrumento para mudança da forma tradicional de prestação da assistência, através do fortalecimento da Atenção Primária à Saúde (APS) ${ }^{2}$. Este programa se expandiu em questões numéricas com ampliação quantitativa e geográfica da cobertura, verificando-se uma tendência temporal de crescimento na implantação de Equipes de Saúde da Família (EqSF) no Nordeste brasileiro, e sua forte correlação com os indicadores sociais e de saúde ${ }^{3}$.

Segundo Macinko, Guanais e Souza ${ }^{4}$, com a instituição do PSF houve também uma diminuição considerável na taxa de mortalidade infantil associada a uma maior cobertura do Programa nos estados brasileiros, juntamente com o aumento da cobertura da imunização e ações de combate e tratamento da diarreia e infecções respiratórias agudas, se mostrando como uma importante ferramenta para o declínio da mortalidade infantil no Brasil, embora não seja o único instrumento neste processo.

Em 2006, com a Portaria no 648/GM, é aprovada a Política Nacional de Atenção Básica (PNAB), que estabeleceu a revisão de normas e diretrizes para a organização deste nível de atenção do PSF e propôs a mudança de nomenclatura de Programa para Estratégia Saúde da Família $^{5}$. Com a revisão das normas e diretrizes para a organização da Atenção Básica, a partir da promulgação da Portaria 2.488 de $2011^{6}$, e da Portaria 2.436 de 20171, que aprova uma nova Política de Atenção Básica, a ESF ainda continua a propor a substituição da forma tradicional da assistência a partir do desenvolvimento de ações pela equipe multiprofissional centradas na família e na comunidade, com atividades realizadas de acordo com o diagnóstico situacional, buscando, assim, a promoção do cuidado individual e coletivo, a partir da efetivação de uma assistência integral, contínua e resolutiva.

Acreditamos que ESF é um marco na incorporação da APS na política de saúde brasileira. Além disso, a (in)satisfação dos usuários com os serviços da ESF, especialmente na questão da acessibilidade, da interação equipe/usuário e do desenvolvimento de ações resolutivas é de grande relevância para o planejamento dos serviços ${ }^{2}$.

A resolubilidade pode ser definida como o desenvolvimento de ações, a partir do uso de diferentes tecnologias e abordagens de cuidado individual e coletivo, capazes de identificar e intervir nos riscos, necessidades e demandas de saúde da população, buscando garantir a solução de problemas de saúde dos usuários ou encaminhando-os para outros níveis de atenção à saúde quando necessário ${ }^{1}$.

Deste modo, a resolubilidade diz respeito a ações em saúde que promovem eficácia e eficiência, que alcançam os resultados desejados tanto pela população como pelos gestores e trabalhadores, envolvendo assim, serviços, equipamentos, tecnologias leves, duras e leveduras, conformação de redes e articulação das existentes, tendo como resultado a resolução do problema apresentado ${ }^{7}$.

Entendemos, portanto, que com o desenvolvimento de ações resolutivas, a integralidade, enquanto princípio do SUS poderá ser efetivada, possibilitando, na maioria das vezes, uma maior satisfação do usuário com a assistência recebida e evitando a sua peregrinação pelos serviços de saúde públicos e particulares em busca de uma assistência que atenda a necessidade que apresenta.

Por conseguinte, este estudo teve como objetivos: compreender o(s) significado(s) da resolubilidade na assistência à saúde na perspectiva dos usuários da Estratégia Saúde da Família (ESF) de um município baiano e identificar a(s) possíveis dificuldade(s) e/ou facilidade(s) encontrada(s) pelos mesmos, para a resolubilidade do problema de saúde que apresenta.

\section{Metodologia}

Trata-se de um estudo qualitativo, que foi realizado em três Unidades de Saúde da Família (USF) do município de Filadélfia-Bahia. Traçamos os seguintes critérios de seleção das USF que 
foram pesquisadas: possuir equipe mínima completa (médico, enfermeiro, técnico ou auxiliar de enfermagem, agentes comunitários de saúde) e estar em funcionamento há mais de um ano.

Os participantes deste estudo foram dez usuários pertencentes à área de abrangência das USF há pelo menos um ano, escolhidos aleatoriamente, que possuíam idade igual ou superior a 18 anos, os quais são identificados nos fragmentos das suas falas de acordo com a ordem que foram entrevistados, por exemplo: Ent. 1, Ent. 2. O quantitativo foi determinado a partir do critério de saturação, ou seja, quando as compreensões, explicações e sentidos atribuídos pelos sujeitos começaram a ter uma regularidade de apresentação nos dados coletados $^{8}$. Salientamos que os dados começaram a se repetir a partir da oitava entrevista.

Utilizamos como técnicas de coleta de dados a entrevista semiestruturada e a observação sistemática. A coleta de dados foi realizada no período de sete de dezembro de 2016 a dois de fevereiro de 2017, após aprovação no Comitê de Ética em Pesquisa da Universidade do Estado da Bahia (UNEB), no dia 16 de novembro de 2016, segundo parecer $\mathrm{n}$ 은 1.830.484/ 2016 (CAAE 58746916.4.0000.0057).

As entrevistas foram realizadas na sala de reunião das USF que atendiam aos critérios estabelecidos, após os entrevistados serem abordados na sala de espera e aceitarem participar da pesquisa. Após a leitura, autorização e assinatura do Termo de Consentimento Livre e Esclarecido (TCLE) as entrevistas e observações eram iniciadas de acordo com um roteiro específico. As entrevistas foram gravadas e posteriormente transcritas, enquanto as anotações das observações eram realizadas após sair da unidade onde a coleta era realizada.

Neste estudo, escolhemos como método de análise dos dados, a Técnica de Análise de Conteúdo Temática, a qual "diz respeito a técnicas de pesquisa que permitem tornar replicáveis e válidas inferências sobre dados de um determinado contexto, por meio de procedimentos especializados e científicos" ${ }^{\text {9:303. }}$.

Usamos as etapas propostas por Minayo, Deslandes e Gomes ${ }^{8}$. Realizamos, num primeiro momento, a organização do material, a partir da leitura flutuante das entrevistas, tomando contato exaustivo com o material e com seu conteúdo. Na segunda etapa, o material foi explorado, a partir da sua codificação, ou seja, a transformação dos dados, realizada através de recortes e associações, usando para isso um quadro onde foi feita a síntese horizontal e a vertical. Por fim, fizemos o tratamento dos resultados e interpretação, na qual os dados coletados foram classificados em duas categoriais e posteriormente realizamos as deduções a partir dos recortes e agrupamentos elaborados.

Assim, as categorias que emergiram a partir da análise dos dados, apresentadas em maiores detalhes nos Resultados e discussão, foram:

- $\quad$ Significados de resolubilidade para os usuários da Estratégia Saúde da Família;

- $\quad$ Resolubilidade na Estratégia Saúde da Família: dificuldades $x$ facilidades.

\section{Resultados e Discussão}

Significados de resolubilidade para os usuários da Estratégia Saúde da Família

De acordo com o Ministério da Saúde ${ }^{7}$, resolubilidade consiste em, quando uma pessoa vai à busca de atendimento ou quando surge um problema de abrangência coletiva sobre a saúde, o serviço correspondente esteja capacitado para enfrentá-lo e resolvê-lo até o nível da sua competência.

Segundo a Política Nacional de Atenção Básica ${ }^{1,6}$, a Atenção Básica é o primeiro nível de cuidados à saúde e deverá desenvolver ações resolutivas, sendo coordenadora do cuidado e ordenadora das redes. Seus fundamentos e diretrizes consistem em resolver a grande maioria dos problemas de saúde demandados pela população - cerca de $85 \%$. Deste modo, acreditamos que esse nível de atenção desenvolvendo essas funções - organização, resolução e coordenação - poderá obter a resolubilidade ou estará bem perto dela.

O significado de resolubilidade na assistência à saúde apresentado por alguns usuários em suas falas evidencia certa semelhança com a PNAB, pois estes consideram que resolubilidade na ESF é justamente a assistência em todos os níveis da saúde incluindo consultas, exames, encaminhamentos, entre outros procedimentos:

[...] é ser assistido desde problemas simples $e$ em todas as complexidades [...] é isso, porque, muitas das vezes a gente não consegue resolver o problema, mais pelo menos nós fomos atendidas, e se não resolver aqui manda para outro lugar (Ent.3).

[...] muitas vezes é não ter solução para um problema de saúde e achar quem the ajude 
a melhorar, aconteceu isso comigo há uns anos atrás, eu tive um problema no útero, $e$ eu não tinha condições de ir para salvador e eles ajeitaram tudo, eu não gastei nada (Ent. 7).

[...] eu acho assim, para mim é quando a gente vai procurar ajuda na assistência social ou na secretaria de saúde, é quando vai pra resolver alguma coisa, por exemplo, se for para marcar um exame é para resolver pra mim, porque eu não sei resolver (Ent.10).

Percebemos que a resolubilidade para estes entrevistados significa encontrar solução para as suas necessidades de saúde nos diversos serviços, desde a Atenção Básica, até a média e alta complexidade. Deste modo, notamos que o fato de obter uma resposta positiva e/ou o encaminhamento necessário proporciona certa satisfação ao usuário, mesmo que o problema não seja resolvido na unidade ou no município onde reside.

A produção da saúde é dependente de vários fatores, dentre esses estão o estabelecimento de redes, vínculos e corresponsabilidade entre usuário-profissional, o que implica diferentes ações, saberes e práticas, almejando sempre uma atenção integral, resolutiva e humanizada, garantindo assim satisfação dos sujeitos nas ações desenvolvidas na $\mathrm{ESF}^{7}$.

Neste sentido, notamos através da observação sistemática que os profissionais de fato contatam a rede de saúde do município e de outras cidades com um nível de complexidade maior para resolver os problemas apresentados pelos pacientes, quando estes não têm a resolução devida na unidade.

Por conseguinte, comungamos com Turrini, Lebrão e $\operatorname{Cesar}^{10}$, pois acreditamos que a resolubilidade é uma das maneiras de se avaliar os serviços de saúde a partir dos resultados positivos ou negativos que são obtidos pelos usuários no atendimento.

De acordo com Costa et al. $^{11}$, a resolubilidade das ações de saúde na ESF está diretamente ligada ao trabalho efetivo da equipe multiprofissional, a qual deve procurar solucionar os problemas individuais e coletivos demandados pela comunidade de modo a garantir um atendimento acolhedor e responsável.

A valorização da presença dos profissionais de saúde na ESF, especificamente do médico, é perceptível na fala dos usuários, os quais consideram que resolubilidade é ter este profissional na unidade, como apresentado nas seguintes falas:

[...] ter mais médico, né (Ent. 5).

[...] o que é solução na saúde? A solução é médico! [...] é médico, é remédio, é isso (Ent.6).

[...] é ter as unidades funcionando adequadamente [...]. Solução em palavras claras é chegar à unidade ou no hospital e ter médico, enfermeiro, técnico atendendo $e$ as unidades funcionando adequadamente para atender a população em geral (Ent. 10).

A presença do médico na unidade é apresentada por esses três entrevistados como sinônimo de resolubilidade. Contudo, o Ent. 6 acrescenta que é necessário também "o remédio" para garantir o tratamento. Além disso, o Ent. 10 afirma que resolubilidade é ter as unidades funcionando adequadamente e para isso é necessário a existência dos profissionais que compõem a equipe.

Assim, notamos o prestígio e a importância que o profissional médico apresenta para os usuários dos serviços de saúde, como explicitado nestas falas, o que nos faz lembrar uma assistência voltada para o modelo médicohegemônico.

Estudo realizado por Esmeraldo et al $^{12}$ demonstrou a coexistência da ESF e do modelo biomédico tradicional, com práticas assistencialistas muitas vezes baseadas nas concepções desse modelo hegemônico. Compreendemos que esse modelo é valorizado não só pela população assistida, mas também, pela mídia, pelos médicos e políticos, caracterizado pela centralização na clínica e no atendimento à demanda espontânea.

A partir da nossa observação nas três USF, percebemos que o médico era realmente o profissional mais procurado pelos usuários para atendimento, o que gerava uma demanda maior para este trabalhador e certa dificuldade para marcação de consultas. Notamos ainda, através das reações e falas dos pacientes a sua satisfação com o atendimento prestado pelo médico. Além disso, observamos que existe um vínculo entre o usuário e o profissional, e apesar do foco estar na doença, eles procuram solucionar o problema de saúde apresentado pelo usuário a partir do diálogo e da busca pela melhor opção de tratamento.

Entendemos que a resolubilidade é uma resposta satisfatória que o serviço de saúde 
oferece ao usuário diante das necessidades e demandas de atenção à saúde que apresenta quando vai em busca de ações que tragam uma solução ao seu problema ${ }^{13}$. Deste modo, os Ent. 2, 4 e 9 definem resolubilidade como solução de seus problemas de saúde, sendo estes um leque de opções, apesar do Ent. 9 ainda acrescentar a presença do médico como necessária para que isto aconteça:

Resolubilidade! É tipo solução? [...] é importante né, é importante! [...] é muito importante resolver nossos problemas (Ent. 2).

[...] é a gente vim aqui e resolver o problema né, um problema da saúde né (Ent. 4).

[...] ter solução né, é resolver os problemas né. [...] assim, quando a gente vem aqui e quer um médico e a agente encontra, ou outra coisa que a gente precisa e encontra (Ent. 9).

Acreditamos que ter acesso a um serviço com ações resolutivas e que ofereça o resultado almejado, proporciona satisfação ao usuário e quando isso não ocorre é preciso a formulação de práticas mais condizentes com as suas demandas, de modo a atendê-las.

E esse resultado alcançado pode ser apenas um atendimento, estando esse marcado ou não, mas o simples fato de conseguir uma consulta ou uma informação que lhe direcione para uma resposta positiva já é vista como resolubilidade, como apresentado pelo Ent. 1:

\section{[...] Vim aqui e ser atendida (Ent.1)}

Concordamos com Turrini, Lebrão e Cesar ${ }^{10}$ que a resolubilidade vai muito além de um diagnóstico, uma prescrição medicamentosa e a cura da doença, ela pode ser obtida por meio de um atendimento acolhedor e responsável, a partir das tecnologias disponíveis.

Notamos que as tecnologias leves, que são aquelas ligadas à relação profissional de saúde/ usuário, se fazem necessárias, muitas vezes, para a promoção da resolubilidade e poderão ser desenvolvidas por toda a EqSF, de modo a garantir a resolução dos problemas apresentados pelo usuário e a sua satisfação.

Acreditamos que a EqSF deverá realizar ações diagnósticas e terapêuticas, prevenção de doenças e promoção da saúde, com vistas a dar uma resposta às necessidades de saúde apresentadas pelo usuário e garantir a resolubilidade nos serviços oferecidos ${ }^{1}$.

Percebemos que nas unidades pesquisadas os profissionais têm caminhado no sentido de efetivar essas atividades preconizadas pelo Ministério da Saúde no desenvolvimento de suas ações. Neste processo, vislumbramos facilidades e dificuldades que contribuem ou interferem para a promoção da resolubilidade, como discutiremos a seguir.

Resolubilidade na Estratégia Saúde da Família: dificuldades $x$ facilidades

A análise da resolubilidade vai depender de quem avalia e do que é avaliado. Assim, nesse processo devem ser considerados aspectos referentes à demanda, à satisfação do usuário, às tecnologias dos serviços de saúde, à existência de um sistema de referência e contrarreferência articulado, à acessibilidade aos serviços, à formação dos recursos humanos, às necessidades de saúde da população, à adesão ao tratamento, aos aspectos culturais e socioeconômicos, entre outros $^{10}$.

Podemos ter várias definições sobre o que é resolubilidade na ESF a partir do olhar daqueles que realizam ações para sua efetivação - os profissionais de saúde - ou dos que buscam a sua implementação para solução de seus problemas - os usuários.

Acreditamos que o usuário como avaliador dos serviços poderá expressar de maneira mais fiel suas experiências e definir com mais exatidão o que é resolubilidade. Deste modo, buscamos nos parágrafos seguintes discutir as principais dificuldades e/ou facilidades identificadas nas falas e observações para o alcance da resolubilidade.

Para os Ent. 1 e 3 uma das dificuldades para a resolubilidade dos problemas perpassa pela demora em receber o resultado de exames, como o preventivo, e em conseguir atendimento com o profissional médico:

Não ter vagas para o médico quando nós precisamos, demora na entrega do preventivo, fora isso eu resolvo meus problemas, graças a Deus (Ent. 1).

[...] tem os preventivos que demora de chegar, [...] ah, outra dificuldade é as visitas domiciliares do médico, às vezes a gente precisa e eles não vão (Ent.3).

Notamos que algumas dificuldades encontradas pelos usuários estão relacionadas à demora na entrega dos resultados dos exames 
citopatológicos, o que poderá causar certa ansiedade nas mulheres. Outro limite descrito para a promoção da resolubilidade foi a obtenção do atendimento médico, seja no consultório ou na visita domiciliar o que reforça a relevância dada a este profissional na atenção à saúde, como discutido anteriormente. Salientamos que as visitas domiciliares se constituem em uma atividade importante a ser desenvolvida pelos profissionais da ESF, pois pode aproximá-los da realidade vivenciada pelos usuários, contudo tem funcionado muitas vezes com dificuldade, frente à falta de transporte, bem como a falta de interesse de alguns trabalhadores em realizá-las.

Estudo realizado por Moimaz et $\mathrm{al}^{14}$ demonstra que a não resolubilidade na assistência à saúde está atrelada, entre outras razões, ao agendamento das consultas médicas e ao tempo que se leva do agendamento até a consulta, demora para a entrega dos exames ou até para a sua realização, não especificando o tipo. O autor ainda menciona a falta de medicação, principalmente aquelas de uso contínuo pelo paciente, causando insatisfações.

A ausência de medicamentos na farmácia básica foi apresentada pelos entrevistados enquanto um aspecto que limita a promoção da resolubilidade, pois os usuários que possuem alguma patologia crônica e que necessitam de tratamento continuado e até mesmo aqueles que utilizam fármacos esporadicamente consideram importante a sua disponibilização de maneira regular e em quantidade suficiente para atender a demanda da população:

Assim, às vezes falta remédio e eu que sou dependente às vezes tenho que comprar, mas isso já é culpa do governo que não manda direito (Ent. 3).

[...] há, os remédios da pressão e da diabetes e outros é difícil ter, isso para mim é uma grande dificuldade, porque tenho que comprar inclusive estou indo aqui sem nada, não tem nenhum do que o médico passou (Ent. 6).

De maneira divergente aos Ent. 3 e 6, o Ent. 8 em sua fala afirma que o que facilita resolver $o$ seu problema é ter a medicação na USF, salientando, contudo, que isso ocorre em alguns momentos:

As facilidades? É, em alguns momentos nas unidades sempre a medicação que a população necessita encontra, as facilidades que eu lembro é essa (Ent. 8).

Durante a coleta de dados pudemos observar que a grande maioria dos usuários que saíam dos consultórios médicos com as receitas em mãos não encontravam os medicamentos prescritos na farmácia da USF, causando descontentamento.

A falta de medicamentos na farmácia básica é apontada como uma dificuldade para a resolubilidade nas ações desenvolvidas na ESF em estudos realizados por Vanderlei e Navarette15 e Campos et $\mathrm{al}^{16}$, pois o tratamento é interrompido ou torna-se impossível a continuação da terapia indicada, devido à falta de recursos financeiros dos usuários para comprá-los. Assim, acreditamos ser relevante garantir a disponibilização regular dos medicamentos, de modo a possibilitar a resolução das necessidades que apresenta.

Outra dificuldade para a promoção da resolubilidade na assistência apresentada pelos usuários, diz respeito ao acesso geográfico das USF, como é relatado nas falas dos Ent. 3 e 8:

[...] outro coisa ruim é a distância [...], o povo mesmo lá de minha rua reclama que ficou muito longe, e fica querendo ir para outro posto que é mais perto [...] (Ent. 3).

A dificuldade é que as unidades ficaram de uma certa forma um pouco distantes das casas, pelo menos aqui na zona rural, aí dificulta um pouco [...] (Ent.8).

A proposta da ESF de aproximar a população dos serviços de saúde e criar vínculos com os usuários é de suma importância. Para que isso aconteça acreditamos ser necessário que as USF estejam localizadas de modo a favorecer o acesso geográfico. Observamos que houve há aproximadamente quatro anos, considerando o momento que realizamos a coleta de dados deste estudo, um redimensionamento das áreas de abrangência das USF, sendo que algumas microáreas ficaram distantes da sede da unidade.

Deste modo, a localização geográfica das USF poderá dificultar a acessibilidade dos usuários às unidades, pois quando estas se situam distantes das microáreas adscritas, constituem-se em barreiras de acesso que comprometem a qualidade do serviço e a adesão dos usuários ${ }^{17}$. Além disso, estudo realizado por Ferreira et al. ${ }^{18}$ reforça em seus resultados que a qualidade da ESF é determinada pela dimensão estrutural que diz respeito dentre outros 
aspectos, ao acesso e a localização da unidade de saúde no território.

Acreditamos, portanto, ser necessário o desenvolvimento de estratégias elaboradas juntamente com toda a equipe da ESF e os gestores, de modo a facilitar o acesso aos serviços para a população. Além disso, a consolidação e estruturação das visitas domiciliares poderá proporcionar o aumento da resolubilidade, a partir da ampliação do acesso dos usuários às ações desenvolvidas pelos profissionais da ESF.

É interessante destacarmos que os Ent. 2 e 6 relataram como aspecto facilitador morar perto da unidade, o que possibilita um melhor acesso aos serviços oferecidos pela ESF:

[...] moro perto da unidade e no município tem facilidade de ser atendido e o povo que trabalha aqui atende bem [...] (Ent.2).

[...] ser atendido, ser atendido não é difícil, morar perto também facilita, porque ando meio cansado, $e$ aqui tem bons profissionais [...] (Ent.6).

Além de morarem perto da unidade esses entrevistados citam como facilidade a existência de "bons" profissionais e o atendimento que estes realizam. Observamos que este fato tem possibilitado a construção do vínculo e do acolhimento, os quais são relevantes para a promoção de um atendimento de qualidade.

Portanto, a maneira como se configura a relação usuário-profissional de saúde poderá se constituir enquanto limite para a promoção da resolubilidade, como enfatizam os Ent. 6 e 7:

[...] só teve uma vez que tive uma pequena discussão aqui, mas deixa para lá né (Ent. 6).

[...] só uma funcionária que era meia assim (fez uma careta), mas já está tudo bem (Ent.7).

Os atendimentos baseados na escuta do usuário, no respeito e no acolhimento poderão propiciar o vínculo usuário-serviço de saúde, bem como a resolubilidade das práticas desenvolvidas ${ }^{13}$.

Apesar desses limites apresentados, salientamos que os entrevistados 2, 4, 9 e 10 afirmaram não encontrarem dificuldades para resolverem seus problemas na ESF:

[...] até agora não tive não [...] (Ent. 2).
[...] nunca tive não, sempre fui atendido [...] (Ent. 4).

[...] tudo que eu quis eles me atenderam [...] (Ent.9).

Graças a Deus não, tudo que eu procuro eu consigo (Ent. 10).

Esta situação pode refletir uma real satisfação dos usuários com os serviços oferecidos ou pode ocorrer justamente por estes usuários aceitarem de forma passiva as respostas que Ihes são oferecidas, recebendo assim tudo que lhe é proposto sem questionar.

Tavares et al. ${ }^{19}$ verificaram que o nível de entendimento e informação da população sobre o que é o SUS e os seus direitos em relação à assistência é deficiente e isto se coloca em muitas situações como uma estratégia de (in)visibilidade sobre os direitos dos usuários e deveres do Estado, no papel de gestores e trabalhadores de saúde.

O processo de promoção de práticas resolutivas, apesar de ser permeado pelas dificuldades elencadas pelos usuários em suas falas, apresenta facilidades. Estas dizem respeito ao acesso geográfico e a disponibilidade dos medicamentos na USF como discutido anteriormente, bem como estão relacionadas à maneira como os profissionais de saúde têm atendido os usuários, conforme demonstra as falas a seguir:

Me atendem bem e me dão assistência e respondem minhas perguntas (Ent. 1).

[...] os profissionais atendem bem, eu mesma quando vou nesse médico chega sinto uma paz, e é todos eles [...] (Ent.3).

[...] eu acredito que é chegar e ser bem atendido, ter assim a forma de atender as pessoas né, [...] a gente tem quer ser bem atendido [...] (Ent. 4).

Assim quando eu procuro sou bem servida, não tenho do que queixar, eles marcam tudo para mim, e sempre me orientam o que tenho de fazer (Ent. 10).

Percebemos a importância de um atendimento acolhedor e humanizado e como este faz a diferença para o usuário. Concordamos com Farias et al. ${ }^{20}$, que a prática do acolhimento é uma importante diretriz que possibilita o acesso e a qualificação do processo de trabalho nos cenários dos serviços de saúde, assim como pode reduzir as barreiras relacionadas ao tempo 
de espera e ao mau direcionamento de consultas e facilita a escolha de prioridades e o aumento do vínculo.

Além da realização de um atendimento acolhedor e humanizado, a orientação dada aos usuários pelos profissionais que compõem a ESF é de suma importância para a promoção da resolubilidade na assistência prestada:

[...] bom, a agente comunitária me visita, leva algumas informações, é muito bom, sou bem tratado, que tem que ser mesmo [...] (Ent. 6).

[...] eu tenho a agente de saúde que sempre me informa quando tem algo, esse negócio [...] do glaucoma [...] e tem a recepcionista aqui que me informa também [...] (Ent. 7).

[...] Desde o acolhimento ao atendimento humanizado, seja na unidade ou no hospital, isso facilita bastante, o atendimento que traz informações [...] (Ent. 8).

[...] é quando eles me ajudam a resolver as coisas, porque eu não sei nada, quando me orientam e mandam eu procurar assim, 'assim e assado', aí eu faço [...] (Ent.10).

Acreditamos que a ESF deverá acolher e buscar solucionar os problemas de saúde apresentados pelo usuário seja na própria USF ou encaminhando-o para serviços adequados. Por isso, os resultados do estudo realizado por Carrapato, Castanheira e Placideli $^{21}$ apontam como principais núcleos de significação da qualidade na Atenção Básica o acolhimento, enquanto interação entre profissional e usuário do SUS. Assim, acreditamos que o acolhimento se constitui em um instrumento relevante para a reorganização dos serviços, ao promover a escuta e resolver as demandas dos usuários.

Deste modo, o encaminhamento para serviços de média e alta complexidade, poderá constituir-se em facilidade para a promoção da resolubilidade:

[...] me encaminha para outro lugar, se não for caso de resolver aqui me mandam para outro lugar [...] (Ent.5).

[...] eles me atendem, né, ou me encaminham para onde tenho que ir [...] (Ent.9).

[...] Eu já fiz tratamento em Salvador e eu fui bem atendida aqui, me encaminharam certinho [...] e sempre marcavam tudo lá
[...], tanto para mim como meu marido (Ent.10).

Conforme apresentado por Souza, Ribeiro e Soares $^{22}$, a satisfação do usuário possibilita a avaliação da qualidade funcional do serviço de saúde e ajuda a identificar se suas necessidades foram resolvidas e as melhores formas de prevêlas e atendê-las.

Ao contrário disso, a insatisfação é determinada como uma expressão negativa, ocorrendo quando o usuário se sente descontente com o serviço prestado ou quando não consegue atingir as expectativas e demandas almejadas $^{23}$.

Diante da realidade encontrada para concretização da resolubilidade na ESF, acreditamos ser importante vencer as dificuldades elencadas e fortalecer as facilidades, com vistas a promover a sua efetivação nas ações desenvolvidas, garantir a qualidade da assistência prestada e uma maior satisfação dos usuários com os serviços oferecidos.

\section{Considerações finais}

Neste estudo, o conceito de resolubilidade apresentado pelos usuários está relacionado, muitas vezes, às necessidades e expectativas que estes apresentam. Deste modo, o significado de resolubilidade perpassa pela assistência em todos os níveis da saúde incluindo consultas, exames, encaminhamentos, entre outros procedimentos, bem como pela presença do profissional de saúde na unidade, sendo o médico o profissional mais citado. Além disso, resolubilidade na visão dos usuários é obter solução dos problemas de saúde que apresenta, ou o simples fato de conseguir uma consulta ou uma informação que lhe direcione para uma resposta positiva.

$\mathrm{Na}$ procura da solução de seus problemas de saúde os usuários se deparam com facilidades, que estão relacionadas às ações desenvolvidas pelos profissionais para a garantia da resolubilidade, bem como a localização geográfica da unidade. Contudo, algumas dificuldades para a efetivação de ações resolutivas são citadas pelos usuários como a demora em receber o resultado de exames, conseguir atendimento com o profissional médico, principalmente domiciliar, ausência de medicamentos na farmácia básica, a distância geográfica da USF em relação aos seus domicílios, entre outros.

Acreditamos que a compreensão do significado de resolubilidade da assistência na 
percepção dos usuários, bem como das dificuldades e/ ou facilidades que permeiam a sua garantia é de suma importância para a reorganização e promoção da qualidade dos serviços de saúde. Assim, a partir do olhar destes sujeitos, os profissionais de saúde e gestores poderão avaliar seus processos de trabalho e propor intervenções mais adequadas, em função das necessidades e expectativas sobre o serviço.

Destacamos que as limitações deste estudo estão relacionadas ao fato de ter sido analisada a realidade de um município específico, bem como por se tratar de uma investigação qualitativa, impossibilita a generalização dos fatos investigados. Entretanto, reforçamos que os resultados encontrados poderão ser semelhantes ao vivenciado em outros contextos e colaborar/ estimular o desenvolvimento de estudos, ações e reflexões que colaborem para a resolubilidade na ESF.

\section{Referências}

1. BRASIL, Ministério da Saúde. Política Nacional de Atenção Básica (2017). Brasília: Ministério da Saúde; 2017.

2. BRASIL, Ministério da Saúde. Saúde da Família: avaliação da implantação em dez grandes centros urbanos. Síntese dos principais resultados. Brasília: Ministério da Saúde; 2005.

3. CARVALHO FCD, VASCONCELOS TB, ARRUDA GMMS, MACENA RHM. Modificações nos indicadores sociais da região nordeste após a implementação da atenção primária. Trab. Educ. Saúde. 2019;17(2):1-21.

4. MACINKO J, GUANAIS FC, SOUZA MFM. Evaluation of the impact of the Family Health Program on infant mortality in Brazil, 1990-2002. J Epidemiol Community Health. 2006;60(1):1319.

5. BRASIL, Ministério da Saúde. Política nacional de Atenção Básica (2006). Brasília: Ministério da Saúde; 2006.

6. BRASIL, Ministério da Saúde. Política Nacional de Atenção Básica (2011). Brasília: Ministério da Saúde; 2012.

7. BRASIL, Ministério da saúde. Secretaria de Atenção à Saúde. Humaniza SUS: Documento Base para Gestores e Trabalhadores do SUS. Brasília: Ministério da Saúde; 2010.

8. MINAYO MCS, DESLANDES SF, GOMES R. Pesquisa social: teoria, método e criatividade. 34 . ed. Rio de Janeiro: vozes; 2015.

9. MINAYO MCS. O desafio do Conhecimento: pesquisa qualitativa em saúde. 12. ed. São Paulo: Hucitec; 2010.
10. TURRINI RNT, LEBRÃO ML, CESAR CLG. Resolubilidade dos serviços de saúde por inquérito domiciliar: percepção do usuário. Cad. Saúde Pública. 2008;24(3):663-674.

11. COSTA JP, JORGE MSB, VASCONCELOS MGF, PAULA ML, BEZERRA IC. Resolubilidade do cuidado na atenção primária: articulação multiprofissional e rede de serviços. Saúde Debate. 2014;38(103):733-743.

12. ESMERALDO GROV, OLIVEIRA LC, ESMERALDO FILHO CE, QUEIROZ DM. Tensão entre modelo biomédico e estratégia saúde da família: percepções dos trabalhadores de saúde. Revista de APS. 2017;20(1):98-106.

13. RIOS MO, NASCIMENTO MAA. Produção do cuidado para resolubilidade da Estratégia de Saúde da Família: saberes e dilemas. Rev. Enferm. UFPE on line. 2017;11(Supl9):3542-3550.

14. MOIMAZ SAS, MARQUEZ JAM, SALIBA O, GARBIN CAS, ZINA LG, SALIBA NA. Satisfação e percepção do usuário do SUS sobre o serviço público de saúde. Revista de Saúde Coletiva. 2010;20(4):1419-1440.

15. VANDERLEI LCM, NAVARETTE MLV. Mortalidade infantil evitável e barreiras de acesso à atenção básica no Recife, Brasil. Revista de Saúde Pública. 2013;47(2):379-389.

16. CAMPOS VS, ARAÚJO PO, ARAÚJO BO, ASSIS MMA, ARAÚJO EGO. Saúde da mulher idosa: resolubilidade do cuidado na Estratégia Saúde da Família. Braz. J. of Develop. 2020;6(12):98787-98802.

17. PAULA CC, SILVA CB, TASSINARI TT, PADOIN SMM. Fatores que interferem no acesso de primeiro contato na atenção primária à saúde: revisão integrativa. Rev. Pesq.: Cuid. Fund. Online. 2016;8(1):4056-4078.

18. FERREIRA J, GEREMIA DS, GEREMIA F, CELUPPI IC, TOMBINI LHT, SOUZA JB. Avaliação da Estratégia Saúde da Família à luz da tríade de Donabedian. Av Enferm. 2021;39(1):63-73.

19. TAVARES FL, LAIGNIER MR, SILVA MZ, DAROS RF, LIMA RCD. O SUS que temos e a informação como estratégia de (in)visibilidade. Saúde em Debate. 2003;27(65):405-413.

20. FARIAS DN, RIBEIRO KSQS, ANJOS UU, BRITO GEG. Interdisciplinaridade e interprofissionalidade na Estratégia Saúde da Família. Trab. Educ. Saúde. 2017;16(1):141-162. 21. CARRAPATO JFL, CASTANHEIRA ERL, PLACIDELI N. Percepções dos profissionais de 
saúde da atenção primária sobre qualidade no processo de trabalho. Saúde e Soc. 2018;27(2):518-530.

22. SOUZA PC, RIBEIRO CF, SOARES SS. Satisfação dos usuários da assistência hospitalar: o caso de um hospital público do SUS do estado de Mato Grosso. Rev. Adm. Saúde. 2017;17(69):1-16.

23. ABRAHÃO-CURVO P. Avaliação da Atenção Básica em saúde destacando satisfação e insatisfação na perspectiva dos usuários, com ênfase na integralidade da atenção [dissertação]. Ribeirão Preto: Escola de Enfermagem de Ribeirão Preto. Universidade de São Paulo; 2010.

\section{Endereço para Correspondência}

Mariana de Oliveira Araújo

BR 116, KM 03, Campus Universitário,

Universidade Estadual de Feira de Santana -

Feira de Santana/BA, Brasil

CEP: $44031-460$

E-mail: mariana-enf@hotmail.com

Recebido em 13/01/2020

Aprovado em 27/03/2021

Publicado em 10/05/2021 